\title{
Análisis de contenido analógico: una aplicación de la hermenéutica analógica para el análisis de contenido curricular
}

\author{
Analogic content analysis: an application of analogic hermeneutics to curricular content analysis \\ David Sebastián Contreras Islas \\ DOI: https://doi.org/10.24215/18537863e071
}

Ciencias de la Salud de la Universidad Autónoma

Metropolitana, unidad Iztapalapa, México

david.contreras@hu-berlin.de

Recepción: 24 Agosto 2019

Aprobación: 17 Febrero 2020

\section{Resumen:}

El análisis de contenido analógico (ACA) combina los análisis de contenido con la hermenéutica analógica, permitiendo jerarquizar la validez de las categorías analíticas e integrar varios niveles interpretativos dentro de un mismo ejercicio. El objetivo de este artículo es exponer los alcances y limitaciones de ACA, tomando como ejemplo el análisis de la bioética en el currículum de la División de Ciencias Biológicas y de la Salud en la Universidad Autónoma Metropolitana, donde ACA facilitó realizar inferencias sobre el contexto de producción de los textos, el ejercicio de la práctica docente, y establecer horizontes para orientar el (re)diseño curricular

Palabras clave: Análisis de contenido, Hermenéutica, Bioética, Educación superior, Currículum.

\section{Abstract:}

Analog content analysis (ACA) combines content analysis with analogue hermeneutics, allowing hierarchy of the validity of analytical categories and integrating several interpretative levels within the same exercise. The objective of this article is to discuss the scope and limitations of ACA, taking the analysis of bioethics in the curriculum of the Division of Biological and Health Sciences at the Autonomous Metropolitan University as an example. There, ACA facilitated making inferences about the context of text production, the actual teaching practice, and establishing horizons to guide future exercises on curricular design

KEYworDs: Content analysis, Hermeneutics, Bioethics, Higher education, Curriculum.

\section{INTRODUCCIÓN}

Las técnicas de análisis de contenido se distinguen por cuantificar de manera sistemática la frecuencia con la que ciertas categorías de análisis (por ejemplo, palabras clave de un campo semántico específico) se presentan en un texto u otra forma de comunicación documentada, con el fin realizar inferencias desde el contenido hacia el contexto (Hernández Sampieri, 2006; Krippendorff, 1990; Mc Kernan, 2001; Piñuel Raigada, 2002). Este proceso implica una tarea hermenéutica, interpretativa, que acompaña la definición de las categorías y la selección de los observables, así como los constructos analíticos necesarios para acceder a elementos de interés que pueden ocultarse por debajo del plano literal del texto. No obstante, esta dimensión hermenéutica no suele abordarse de forma explícita en el diseño metodológico, por lo que se pierde la posibilidad de acceder a dimensiones cualitativas del proceso analítico que son clave tanto para considerar aspectos específicos del contexto de producción de los textos ${ }^{1}$ como para valorar críticamente la validez de sus resultados. En lo sucesivo se procura mostrar cómo el acto de incorporar esta dimensión hermenéutica al análisis de contenido,

\section{NotAS DE AUTOR}

Investigador asociado al departamento de Ciencias de la Salud de la Universidad Autónoma Metropolitana, unidad Iztapalapa, Ciudad México, México, y candidato a doctor por el departamento de Ciencias de la Educación de la Humboldt Universität zu Berlin, Berlín, Alemania. 
especialmente cuando se parte de una hermenéutica analógica (HA), permite distinguir varios niveles de validez interpretativa dentro de un mismo ejercicio analítico, lo que abre nuevas posibilidades que podrían ser de gran interés para la investigación, la innovación y el diseño curriculares (entre otros campos).

El Análisis de Contenido Analógico (ACA) -que resulta de esta novedosa aplicación de la HA- amplía el alcance y mejora la flexibilidad de las técnicas de análisis de contenido tradicionales. En el campo de la investigación educativa puede devenir una herramienta especialmente adecuada, tanto para valorar la integración de contenidos curriculares transversales como para estudiar la asimilación al currículum de temáticas como la sustentabilidad, la bioética, la formación ciudadana o el pensamiento crítico, las cuales, por su origen reciente y su naturaleza interdisciplinaria y compleja, resultan difíciles de aprehender para las técnicas clásicas de análisis de contenido. Además, ACA posibilita incorporar elementos cualitativos y cuantitativos en un mismo ejercicio analítico.

Con el objetivo de ilustrar alcances y limitaciones de esta propuesta, se expone el desarrollo y los resultados de ACA respecto a los contenidos de bioética en el currículum formal de las licenciaturas de la División de Ciencias Biológicas y de la Salud (DCBS) en las unidades Iztapalapa y Xochimilco de la Universidad Autónoma Metropolitana (UAM) dela Ciudad de México, estudio conducido durante el primer semestre de 2018. Por medio de esta exposición se busca ejemplificar algunos de los alcances y limitaciones de ACA, así como destacar la flexibilidad que obtiene al organizar las categorías analíticas en jerarquías de validez, lo que facilita el acceso a aspectos del contexto de producción y de interpretación de los textos, que resultan valiosos para realizar ejercicios críticos de planeación y (re)diseño curricular en la universidad. No obstante, se reconoce que la incorporación de categorías analíticas que demandan lecturas hermenéuticas más alegóricas puede presentar retos para la automatización de los procesos de análisis. El artículo concluye con una invitación a la comunidad académica para explorar otras aplicaciones de esta técnica, a fin de profundizar en el entendimiento de sus alcances y limitaciones.

\section{HERMENÉUTICA Y HERMENÉUTICA ANALÓGICA}

En sentido clásico, la hermenéutica comprende el arte de interpretación de los textos. Para autores como Grondin (2002; 2008), sus orígenes se remontan a los ejercicios de reinterpretación alegórica de los mitos griegos llevados a cabo por los estoicos y posteriormente por Filón de Alejandría, cuya escuela sienta un importante precedente en la alegoresis de los textos del Antiguo Testamento, en contraposición con las lecturas literalistas preferidas por la escuela de Antioquia. De acuerdo con Beuchot, dicha tensión entre la interpretación alegórica y la literal perduró durante la Edad Media, y llegó a volverse una especie de rasgo recurrente a lo largo de la historia de la hermenéutica (Beuchot, 2015).

Ya en la época moderna, con Schleiermacher y Dilthey, la hermenéutica deviene una "reflexión metodológica sobre la pretensión de verdad y el estatuto científico de las ciencias del espíritu" (Grondin, 2008, p. 18). Sin embargo, no es hasta el Siglo XX que alcanza su auge como teoría filosófica, gracias al modelo ontológico de Heidegger, y posteriormente a los trabajos Gadamer, Ricœur y Derrida, entre otros, que elevan el estatus de la hermenéutica al de una filosofía universal de la comprensión (Beuchot, 2015; Grondin, 2008).

Aunque la visión metodológica de la hermenéutica haya sido trascendida durante el giro filosóficoontológico del S. XX, dentro del campo de la metodología de las ciencias sociales, la hermenéutica puede considerarse aún como una forma de fenomenología interpretativa que integra la dimensión temporal e histórica a la fenomenología descriptiva de Husserl. Como tal, suele agruparse entre las metodologías de análisis cualitativo (Sloan $\&$ Bowe, 2014). ${ }^{2}$

Para Beuchot, la historia de la hermenéutica se ha caracterizado por oscilar entre las posturas que defienden una interpretación univocista (textual o literal) y aquellas que se inclinan por una lectura equivocista (alegórica o metafórica) de los textos. No obstante, señala, ambos extremos despojan de sentido a la actividad hermenéutica, por lo que deben ser evitados. Para ello, resulta esencial poner el texto en su contexto: 
interpretar es poner un texto en su contexto. En el acontecimiento hermenéutico o acto interpretativo interviene, en primer lugar, un texto, que es lo que se va a interpretar. Pero ese texto supone un autor. Y, asimismo, un lector o intérprete que es quien lo va a interpretar (Beuchot, 2015, p. 14).

Para buscar este punto medio, Beuchot se apoya en el concepto de analogía, cuyo origen se remonta a los trabajos matemáticos de los pitagóricos, y que Aristóteles supo aplicar a los distintos campos de la filosofía (Beuchot, 2006). La analogía es la proporción, aquello que resulta adecuado o suficiente. Se relaciona, pues, con los conceptos aristotélicos de mesotes y phronesis. Aplicada a la hermenéutica como metodología de la interpretación de los textos, la analogía remite a la paráfrasis mediada por la phronesis, que busca la justa proporción entre la literalidad y la alegoría, entre lo unívoco y lo equívoco.

Al buscar un punto medio entre los extremos del univocismo y el equivocismo, la actividad hermenéutica puede entenderse como una forma de virtud en sentido aristotélico. En este sentido, Beuchot habla de una virtud hermenéutica que comprende "la interpretación cualificada, que se forma por la experiencia y el estudio, sobre todo por la práctica interpretativa sobre los muchos textos” (Beuchot, 2006, p. 240). Adquirida a través del hábito y la práctica, la virtud hermenéutica logra evitar los extremos viciosos, poniendo el texto en su contexto de producción, pero también de interpretación, para demarcar la validez de las posibles lecturas.

De esta forma, Beuchot propone una hermenéutica construida con base en el concepto pitagóricoaristotélico de analogía, entendida como la proporcionalidad virtuosa entre dos extremos: una hermenéutica analógica (HA) que, sin descuidar el sentido literal, incorpore el sentido metafórico o simbólico que pasaría desapercibido en un análisis literalista (Beuchot, 2006).

Al posicionarse en una proporción virtuosa entre la literalidad y la alegoría, entre la metáfora y la metonimia, la HA hace posible "tener más de una interpretación válida, pero sin que todas se nos vuelvan tales" (Beuchot, 2006, p. 246). Más importante: el conjunto de interpretaciones válidas se vuelve jerarquizable para "discernir cuándo una interpretación se empieza a alejar del texto y cuándo ya es francamente errónea” (Beuchot, 2006, p. 246), con lo que se abre la posibilidad de ordenar las interpretaciones dentro de una escala gradual según su grado de univocidad o equivocidad.

La posibilidad jerarquizar las interpretaciones de acuerdo a su grado de validez es quizás uno de los mayores atractivos de la propuesta de Beuchot, que ha encontrado aplicaciones en la investigación dentro de las más diversas disciplinas del área de las ciencias sociales y las humanidades, incluyendo las ciencias de la educación y la pedagogía (Beuchot, 2007; Conde Gaixola, 2001; Conde Gaxiola, 2010; Galindo González, 2010; Palazón Mayoral, 2014; Rivero Weber, 2006; Velazco Gómez, 2006). En nuestro caso, hemos aplicado la HA al análisis de contenido.

\section{ANÁLISIS DE CONTENIDO Y HERMENÉUTICA}

Krippendorff (2004) define el análisis de contenido como "una técnica de investigación para hacer inferencias replicables y válidas desde textos (u otros objetos significativos) hacia el contexto en el que son usados" (p. 18). Originalmente esta técnica se basó en la cuantificación de aspectos recurrentes y fácilmente identificables del contenido manifiesto de comunicaciones escritas, pero ha evolucionado para incluir otras formas de comunicación, así como elementos inmanentes (Domas White \& March, 2006). Aunque se dio a conocer como una técnica cuantitativa (Hernández Sampieri, 2006; Krippendorff, 1990; Mc Kernan, 2001; Piñuel Raigada, 2002), hoy en día existen variantes cualitativas del análisis de contenido (Domas White \& March, 2006; Elo \& Kyngäs, 2007; Krippendorff, 2004; Rourke \& Anderson, 2004).

La principal diferencia entre las variantes cuantitativas y cualitativas radica en la naturaleza deductiva de las primeras (ideal para probar hipótesis en campos relativamente bien estudiados) y la naturaleza inductiva de las segundas (ideal para estudios exploratorios) (Domas White \& March, 2006; Elo \& Kyngäs, 2007). Más allá de esto, ambas variantes comprenden una serie de pasos similares: 
1. Se selecciona el texto o material comunicativo a analizar y se establecen las unidades de análisis. Según el tipo de comunicación, la unidad analítica puede ser una letra, una palabra, una oración, la porción de una página o incluso el número de personas que participan en una discusión.

2. Durante la codificación, las unidades de análisis se ordenan en categorías. En el caso del análisis cuantitativo estas categorías se establecen a priori, con base en el conocimiento previo de la problemática de estudio, mientras que en el análisis cualitativo se van generandodurante la revisión de comunicaciones mediante codificación abierta, a posteriori.

3. Finalmente, se presenta un análisis de las categorías, en el que puede recurrirse a diferentes métodos estadísticos, que dependen de la naturaleza exploratoria, descriptiva o inferencial de la investigación.

Para pasar del texto al contexto y responder las preguntas de investigación, es necesario recurrir a constructos teóricos y analíticos, o bien a reglas de inferencia. Dado que el texto y el contexto son lógicamente independientes y "el investigador transfiere inferencias de un dominio independiente (el texto) al otro (el contexto)" (Domas White \& March, 2006, p. 27), la reproducibilidad de los resultados dependerá de que los constructos teóricos y analíticos empleados se hagan explícitos y sean comprensibles. De lo anterior se sigue, además, que todo análisis de contenido descansa sobre una base hermenéutica, que implica una asimilación de la arquitectura del texto, la traducción de sus enunciados (u otras unidades de análisis) y la búsqueda de sus proposiciones base.

Esta base hermenéutica es especialmente evidente durante la fase de codificación, cuando el investigador se da a la tarea de construir las categorías analíticas con sus respectivos esquemas de codificación. Dado que estos esquemas orientan el proceso interpretativo del investigador o equipo de investigadores, es fundamental que presenten definiciones claras, instrucciones fáciles de seguir y ejemplos inequívocos para promover la confiabilidad y reproducibilidad de los resultados (Domas White \& March, 2006). Desde la teoría de Beuchot, podemos decir que los esquemas deben prevenir un equivocismo excesivo que conduzca a resultados irreproducibles. Al mismo tiempo, sin embargo, se abre la posibilidad de definir intencionalmente esquemas más flexibles, que trasciendan el contenido literal del texto para acceder no solo a los elementos que subyacen a dicha literalidad, y que son, a menudo, indispensables para llevar a cabo inferencias válidas sobre su contexto, ya sea de producción o de interpretación.

Distintas interpretaciones basadas en esquemas distintos tendrán una distinta validez, entendida como "la medida en que un procedimiento de medición representa el concepto previsto, y sólo el previsto" (Neuendorff, 2002, p. 112). La validez puede variar dependiendo tanto de la claridad de los esquemas de codificación como de la complejidad de los elementos que se desea estudiar, así como los constructos analíticos que se emplean para estudiarlos.

Rourke y Anderson (2004) presentan varios ejemplos que ilustran el problema de la validez de los estudios de análisis de contenido en el área de la investigación educativa. En uno de ellos se trataba de estimar la participación y el pensamiento crítico en una discusión en línea por medio del número de veces que los estudiantes contribuían a la discusión y el número de estas contribuciones en las que se formulaban proposiciones a partir de afirmaciones anteriores. La participación aparece como una construcción conceptual mucho menos compleja de observarse y cuantificarse que el pensamiento crítico. El constructo analítico para aprehender la primera (número de veces que los estudiantes aportan a la discusión) es un elemento que se puede observar directamente en la unidad de análisis (foro de discusión) y no presenta, al menos desde la perspectiva de estos autores, mayor ambigüedad para su codificación. En comparación, el segundo concepto es más abstracto, por lo que su aprehensión demanda constructos analíticos más elaborados, que permitan inferirlo a partir de ciertos elementos del contenido manifiesto en las comunicaciones. De esta manera, cabe suponer una mayor probabilidad de ambigüedad durante el proceso de codificación. En consecuencia, podría cuestionarse si las inferencias obtenidas a partir de los resultados del estudio para la participación y para el pensamiento crítico poseen la misma validez. 
Al buscar obtener resultados reproducibles, todo análisis de contenido aspira a la univocidad, sin embargo, se ve limitado por el uso de constructos analíticos más o menos equívocos que permitan pasar del dominio del texto al del contexto. La equivocidad afecta la validez y la reproducibilidad de los resultados. En esta pugna entre la univocidad y la equivocidad la $\mathrm{HA}$ aparece como una alternativa enriquecedora, ya que exige que el intérprete se ubique en su propia situación para reducir la equivocidad, y, conjuntamente, para reconocer la autonomía del sentido del texto, su literalidad.

\section{El ANÁLISIS DE CONTENIDO ANALÓgico (ACA)}

Incorporar un enfoque de HA en los procesos interpretativos que comprenda el análisis de contenido permite integrar información sobre las diferencias de validez de los esquemas de codificación empleados, y generar una escala para distinguir lecturas más alegóricas de otras más literales, lo que posibilita valorar la confiabilidad de los resultados y abrir nuevas oportunidades analíticas. En el núcleo de esta propuesta radica la construcción de metacategorías de validez que, en una escala ordinal, ubiquen las categorías de análisis (o bien, los distintos esquemas de codificación posibles para aproximarse a los observables de cada categoría de análisis) dentro de un continuum entre la lectura literal o unívoca, hasta la interpretación alegórica más equívoca, sin incurrir en posiciones reduccionistas o dicotómicas. Para la construcción de dichas escalas, resulta indispensable tomar en consideración los contextos de producción de los textos, que a menudo se dejan de lado en las técnicas de análisis más tradicionales (Bush et al., 2012).

De acuerdo con su posición dentro de la escala, se asigna un valor hermenéutico (vh) ordinal, numérico y específico para cada categoría analítica, esquema de codificación, o grupo de categorías analíticas (Figura 1). De tal forma, el valor hermenéutico constituye una metacategoría a la que se subordinan las categorías analíticas (Figura 1A), o bien los esquemas de codificación dentro de ellas (Figura 1B), según el grado de univocidad o equivocidad subyacente a cada proceso de interpretación.

Para construir la jerarquía de valores hermenéuticos debe considerase la información disponible sobre contexto de producción de los textos, la complejidad o ambigüedad de los conceptos que desean estudiarse, así como el juicio autocrítico del investigador sobre las limitaciones de sus categorías analíticas. Otros factores que pueden influir en la validez de la interpretación pueden ser la familiaridad entre los contextos de producción e interpretación del texto, e incluso la claridad que (voluntariamente o no) se imprima en los esquemas de codificación (Rourke \& Anderson, 2004).

A través de esta organización en metacategorías, el valor hermenéutico posibilita el empleo de dos o más esquemas de codificación con diferente grado de validez (ya sea por estar más o menos cerca de la univocidad y la equivocidad) para aproximarse a un mismo elemento (o, por ejemplo, para incluir diferentes definiciones de un solo concepto en el mismo análisis). En el caso de la investigación educativa, esta apertura a la pluralidad de observables resulta especialmente útil para el estudio de conceptos novedosos, y, a veces, vagos o controversiales, como sustentabilidad, formación ciudadana o pensamiento crítico, que además pueden encontrarse integrados al currículum escolar bajo múltiples formas, estrategias y matices. Como se verá en nuestro ejemplo sobre el contenido de bioética en los planes de estudio de la UAM, el uso de esquemas de codificación con distintos valores hermenéuticos permite apreciar diferentes formas en las que una misma temática se encuentra integrada al currículum, lo que lleva en el proceso a la identificación de posibles áreas de oportunidad para fortalecerla, así como patrones que resuenan con el desarrollo histórico de la bioética y de la propia institución universitaria. 


\section{ANÁLISIS DE CONTENIDO DE BIOÉTICA EN PLANES DE ESTUDIO}

Durante el primer semestre de 2018 se condujo un estudio de ACA para para explorar elementos de bioética en los planes de estudio de Licenciatura (Tabla 1) en la División de Ciencias Biológicas y de la Salud (DCBS) de la Universidad Autónoma Metropolitana (UAM), Unidades Iztapalapa y Xochimilco. En el caso de Iztapalapa, dicho estudio contaba con un antecedente en 2014 (Contreras Islas et al., 2016), basado en una técnica tradicional de análisis de contenido cuantitativo, que sienta un punto de referencia para comparar el alcance y las limitaciones de ambas técnicas.

Ambos estudios mencionados consideraron una noción de bioética amplia, como la sostenida por Van R. Potter (1971), que incluye las problemáticas propias de la micro, meso y macrobioética, ${ }^{3}$ según las definiciones de Álvarez (2015). En México, esta noción de bioética ha sido adoptada, defendida y desarrollada por autores como González Valenzuela (2009), Linares Salgado (González Valenzuela y Linares Salgado, 2013) o Sagols (2013). La adopción de este enfoque resulta pertinente debido a la diversidad de las Licenciaturas agrupadas dentro de la DCBS de Iztapalapa y Xochimilco (Tabla 1).

Con casi 45 años de existencia, Iztapalapa y Xochimilco son dos de las unidades universitarias más antiguas de la UAM y cuentan con Comités de Ética que han promovido la integración de la bioética al currículum de la DCBS desde 2010 y 2015 respectivamente. No obstante, las unidades poseen modelos académicos muy distintos: mientras que Iztapalapa ha mantenido un sistema tradicional con asignaturas independientes, Xochimilco organiza la docencia en un sistema modular, orientado por objetos de transformación que se abordan desde una perspectiva interdisciplinaria y teórico-práctica durante periodos trimestrales. Asimismo, destaca el predominio de licenciaturas del campo de las ciencias biológicas en Iztapalapa, mientras que en el caso de Xochimilco destaca el de las ciencias de la salud (Tabla 1). Las diferencias entre ambas unidades ayudan a resaltar la flexibilidad de ACA para adaptarse a distintos contextos y modelos curriculares, lo que facilita su comparación. 
TABLA 1

Licenciaturas de la DCBS en la UAM Iztapalapa y la UAM Xochimilco

\begin{tabular}{|c|c|c|}
\hline Unidad Académica & Licenciaturas & $\begin{array}{l}\text { Principal enfoque de } \\
\text { bioética }\end{array}$ \\
\hline \multirow{6}{*}{ Iztapalapa } & Biología & Macro \\
\hline & Biología Experimental & Meso, Macro \\
\hline & Hidrobiología & Macro \\
\hline & Ingeniería Bioquimica Industrial & Macro \\
\hline & Ingeniería de los Alimentos & Macro \\
\hline & Producción Animal & Macro \\
\hline \multirow{8}{*}{ Xochimilco } & Agronomía & Macro \\
\hline & Biología & Macro \\
\hline & Enfermeria & Micro, Meso \\
\hline & Estomatología & Micro, Meso \\
\hline & Medicina & Micro, Meso \\
\hline & Medicina Veterinaria y Zootecnia & Macro \\
\hline & Nutrición Humana & Micro, Meso \\
\hline & Química Farmacéutica Biológica & Micro, Meso \\
\hline
\end{tabular}

Fuente: elaboración propia, 25 de noviembre de 2018.

elaboración propia, 25 de noviembre de 2018.

Las unidades de análisis seleccionadas para explorar el contenido de bioética en los planes de estudio de las licenciaturas fueron los programas de sus respectivas unidades de enseñanza-aprendizaje (UEA). ${ }^{4} \mathrm{~A}$ continuación se proponen tres esquemas de codificación con diferente valor hermenéutico:

a) En el extremo univocista se consideró como indicador de un contenido explícito el que un programa de UEA contuviera la palabra bioética, o alguno de sus derivados inmediatos (por ejemplo, bioético, bioéticos, bioéticas). En este caso se asignó un valor hermenéutico de tres $(v h=3)$. Este tipo de contenido se asocia con un contexto global en el que los gremios profesionales del campo de las ciencias de la salud han ejercido presión, a través de diversas instituciones, para hacer de la bioética (entendida como ética médica) un elemento formativo obligatorio.

b) Para el segundo nivel de valor hermenéutico se consideró el hecho de que el ejercicio profesional de las ciencias biológicas y de la salud implica el trabajo con animales de laboratorio, sujetos de investigación, especies silvestres y otros tipos de sistemas biológicos, que, en el contexto mexicano, se encuentran cobijados por marcos normativos que remiten a las tres dimensiones de la bioética. Se consideró también que los comités de ética de ambas unidades hacen referencia explícita a dichos marcos normativos como aspectos a considerar en el ejercicio de la docencia y la investigación. En consecuencia, dado el contexto de producción de las unidades de análisis, conceptos como ética profesional y sus derivados inmediatos (por ejemplo, profesionistas éticos) se tomaron como referencias indirectas de bioética, asignándoles un valor hermenéutico de dos $(v h=2)$. Este tipo de 
contenido remite, además, al proyecto académico original de la UAM, que busca constituirse como una universidad comprometida con los grandes problemas sociales. ${ }^{5}$

c) En el extremo equivocista se consideraron las frases y temáticas de la unidad de análisis que estuvieran relacionadas con una problemática típica de alguna de las tres dimensiones (micro, meso y macro) de la bioética, como indicadores de un contenido implícito, casi invisible. Para orientar el proceso de codificación, se elaboró un listado de estas temáticas con base en el índice temático del libro Diálogos de Bioética, de González Valenzuela y Linares (2013). A este listado se añadió la categoría bioética y género, a fin de incluir una problemática ampliamente trabajada en México por Sagols (2013, 2015). Al dar pie para interpretaciones más alegóricas y equívocas, el contenido relacionado con este listado de categorías (Tabla 2) recibió un valor hermenéutico de uno $(\mathrm{vh}=1)$. Este tipo de contenidos remite a un contexto más actual, en el que los movimientos ecologistas, animalistas y feministas, entre otros, han permeado en los planes de estudio, sin que por ello formen parte de la identidad institucional, o sean promovidos activamente por los gremios profesionales.

Finalmente, se reservó un valor hermenéutico de cero $(v b=0)$ para hacer referencia a aquellas unidades de análisis en las que no se encontró ninguna referencia explícita o implícita a la bioética. A cada unidad de análisis se le asignó el valor hermenéutico más alto encontrado en su contenido. La Tabla 3 resume el significado de los distintos valores hermenéuticos, que para el caso de este análisis componen una escala ordinal del 0 al 3. 
Revista latinoamericana de Metodología de las Ciencias Sociales, 2020, 10(1), Junio-Noviembre, ISS...

TABLA 2

Categorías de bioética para orientar el análisis hermenéutico

Tabla 2: Categorias de bioética para orientar el análisis hermenéutico

Categoría Descripción

Medicina y

sociedad

Aborda temas de justicia en el ámbito médico y su efecto en la sociedad

Decisiones al en general, asi como las relaciones de salud-enfermedad,

inicio y final médico-paciente-sociedad, etc

de la vida

Aborda problemas en torno al control de la natalidad, las técnicas de reproducción asistida, el alargamiento artificial de la vida y o la eutanasia.

Neuroética Se centra en la a posibilidad de afectar la capacidad ética de seres humanos a partir de avances en el campo de las neurociencias.

Bioética y Aborda problemas del campo de la salud y el medio ambiente desde Género una perspectiva de género.

Biotecnología y Se centra en las consecuencias del desarrollo y uso de las biotecnologías bioética para el ser humano, el ambiente y la vida en general.

Bioética y

evolución

Aborda el origen evolutivo de la capacidad moral, no solo en homínidos, sino a través de otras especies, cuestionando la exclusividad de la capacidad ética del ser humano

Herencia,

bioética y

Aporta elementos para reflexionar sobre las obligaciones morales del ser biodiversidad humano hacia el resto de los seres vivientes.

\section{Ecoética y}

Problematiza la relación de los seres humanos con los animales (en las

Zooética relaciones productivas, en la investigación o en los temas de conservación y bienestar animal) y con el medio ambiente, con énfasis en las dimensiones de la sustentabilidad

Tabla 2: Categorias de bioética para orientar el análisis hermenéutico de textos (proyectos de investigación, planes y programas de estudio) de la DCBS. Las categorías se han organizado de manera descendente desde las más cercanas a la microbioética (en la parte superior) hasta las propias de la macrobioética (en la parte inferior). Fuente: elaboración propia en colaboración con el Programa Universitario de Bioética de la UNAM. Fecha de elaboración: 16 de abril de 2018 . 
TABLA 3

Valor hermenéutico

\begin{tabular}{|c|c|c|}
\hline Valor & Interpretación & Descripción \\
\hline 0 & Sin contenido & $\begin{array}{l}\text { El contenido del texto no guarda ninguna relación con la } \\
\text { bioética, o bien guarda una relación demasiado indirecta }\end{array}$ \\
\hline 1 & Contenido implícito & $\begin{array}{l}\text { El contenido del texto guarda establece una relación } \\
\text { directa con alguna de las categorias de bioética. }\end{array}$ \\
\hline 2 & $\begin{array}{l}\text { Contenido } \\
\text { contextual }\end{array}$ & $\begin{array}{l}\text { El contenido del texto hace alusión a otras formas de la } \\
\text { ética que, en el contexto de las ciencias biológicas, } \\
\text { implican bioética. }\end{array}$ \\
\hline 3 & Contenido explícito & $\begin{array}{l}\text { El texto hace referencia a la bioética o alguna de sus } \\
\text { categorias de manera literal }\end{array}$ \\
\hline
\end{tabular}

Tabla 3: Jerarquía y significado del valor hermenéutico. Fuente: elaboración propia. Fecha: 19 de abril de 2018

Se analizó un total de 514 programas de UEA, de los cuales 384 pertenecieron a la unidad Iztapalapa y 130 a la unidad Xochimilco. La diferencia entre el número de programas deriva del modelo académico de cada unidad, que da lugar a un promedio de 64 UEA o asignaturas en Iztapalapa frente a 15 UEA o módulos en Xochimilco. 192 elementos de esta muestra contaron con un valor hermenéutico mayor o igual a uno $(v h \geq 1)$ indicando algún contenido de bioética. De estos, 77 pertenecieron a Iztapalapa, donde predominó la categoría de ecoética y zooética con 51 casos. En Xochimilco se encontraron 75 UEA con contenido de bioética, de los cuales 53 se relacionaron con la categoría de medicina y sociedad. Estos resultados sugieren una tendencia de los programas de estudio la unidad Xochimilco a incluir contenidos propios de la microbioética y mesobioética, lo que resulta coherente con la inclinación de sus licenciaturas hacia el campo de las ciencias de la salud. En Iztapalapa, el predominio de contenidos propios de la macrobioética es consistente con su inclinación a las ciencias biológicas.

\section{ALCANCES Y LIMITACIONES DE LA PROPUESTA}

En esta sección presentaremos una selección de resultados del estudio antes mencionado, con el objetivo de ilustrar los alcances y las limitaciones de ACA. Los resultados más relevantes para esta discusión se condensan en la Tabla 4 con los siguientes indicadores:

- UEA-total. Total de UEA en cada plan de estudios. Relevante debido a las diferencias de los modelos académicos de Xochimilco e Iztapalapa.

- UEA-bioética. Total de UEA con contenido de bioética. Cuenta total unidades de análisis con $v h$ $\geq 1$ en un plan de estudio.

- \%-UEA-bioética. Porcentaje de unidades de análisis con $v h \geq 1$ en un plan de estudios.

- Cat. Dom. Categoría dominante. La moda las categorías de bioética (Tabla 2) para las unidades de análisis con $v b \geq 1$ en un plan de estudios.

- Cont. Dom. Contenido dominante. La moda del valor hermenéutico (Tabla 3) para las unidades de análisis con $v h \geq 1$ en un plan de estudio.

- Cont. Gen. Contenido general. División de la suma el valor hermenéutico de todas las unidades de análisis de un plan de estudios entre UEA-total. Un valor cercano a 3 indica un contenido explícito 
en la gran mayoría de las UEA. Un valor cercano a 1 indica un predominio del contenido implícito. Un valor cercano a 0 indica ausencia de este tipo de contenido.

Los valores de UEA-bioética y \%-UEA-bioética reflejan un contenido diferenciado de bioética en los distintos planes de estudio. Para el caso de Iztapalapa, estos valores son proporcionales a los reportados por Contreras Islas et al. (2016), obtenidos mediante un análisis de contenido cuantitativo, basado en la búsqueda de palabras clave. Dicho estudio encontró una diferencia de casi 30 puntos porcentuales entre el contenido de los planes de estudio de las licenciaturas como Hidrobiología o Producción Animal, en comparación con los de las licenciaturas en Biología Experimental o Ingeniería Bioquímica Industrial. Al emplear ACA, se encontró una diferencia de más de 20 puntos porcentuales entre los mismos grupos de licenciaturas. La coincidencia de proporción en los contenidos encontrados sugiere que ACA posee, al menos, un alcance similar al de las técnicas de análisis de contenido tradicionales. 
David Sebastián Contreras Islas. Análisis de contenido analógico: una APlicación de la hermenéutic...

TABLA 4

Análisis del contenido de bioética en los planes de estudio de licenciatura de la DCBS de Iztapalapa y Xochimilco

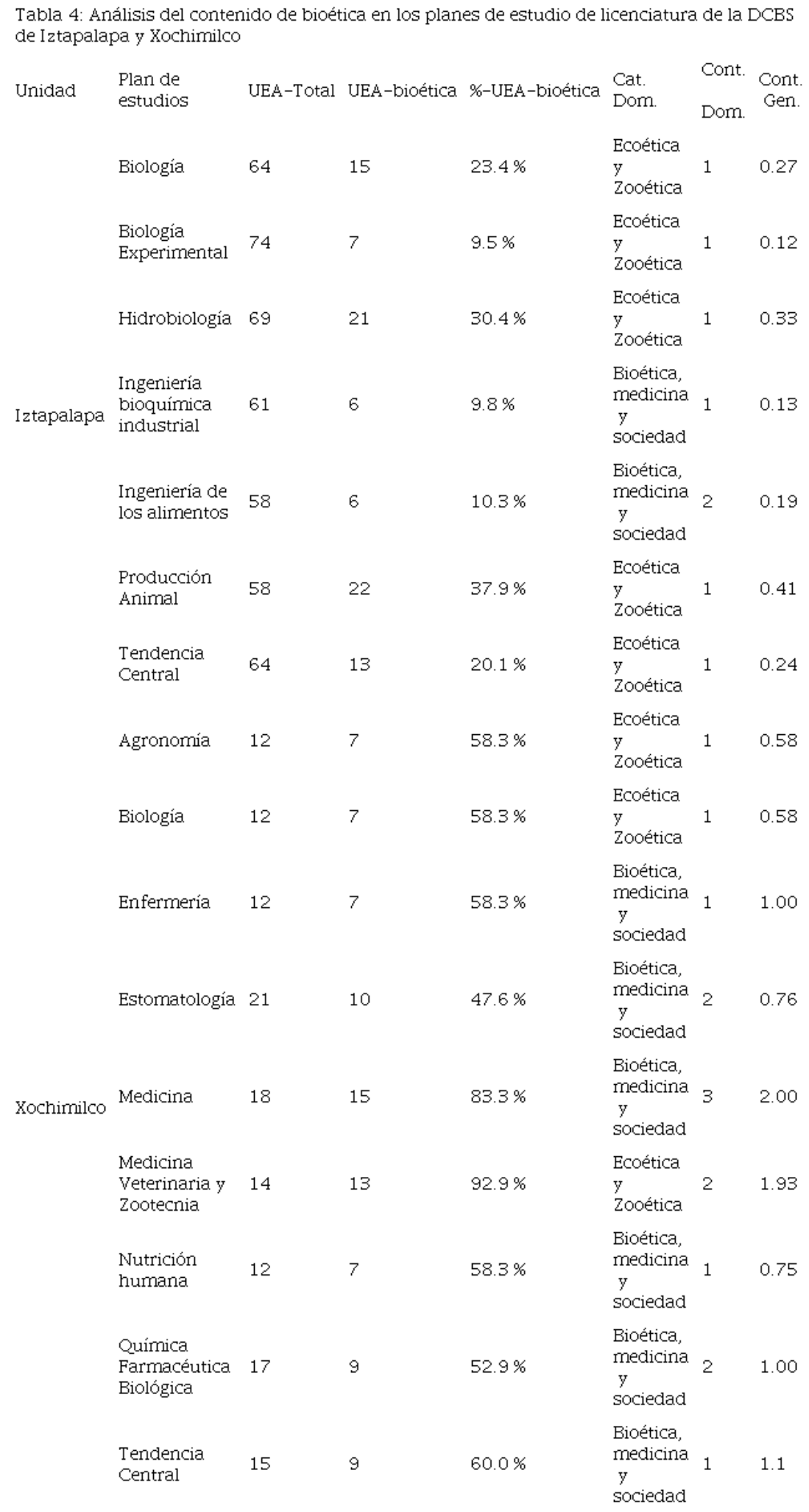

Tabla 4: análisis del contenido de bioética en los planes de estudio de licenciatura de la DCBS en las Unidades Iztapalapa y Xochimilco de la UAM. La Tendencia Central presenta la media aritmética para todos los indicadores excepto Cat. Dom. y Pres. Dom., donde indica la moda. Fuente: elaboración propia. Fecha: 24 de abril de 2018 
ACA, sin embargo, no se limita a una comparación cuantitativa del contenido encontrado en los planes y programas de estudio. A través del valor hermenéutico, permite acceder a dimensiones cualitativas que permanecerían veladas para un análisis de contenido cuantitativo tradicional. Así, por ejemplo, el contenido dominante, en conjunto con la información recopilada en cuanto al contexto de producción de los textos permite inferir aspectos relevantes sobre la calidad con que la bioética se presenta en cada plan de estudios. Un contenido dominante de 1 , que es la más frecuente en ambas unidades, hace dudar de que los elementos de bioética en el contenido se estén abordando de manera satisfactoria. Sugiere, asimismo, la ausencia de elementos suficientes dentro de los planes de estudio para suponer que los contenidos encontrados estén siendo abordados en la práctica docente más allá la perspectiva disciplinaria (limitada) de cada plan de estudios en particular. Por ello, deben interpretarse más bien como indicadores de espacios potenciales para robustecer el contenido de bioética en futuras modificaciones al currículum.

A partir de estos datos, parece válido suponer que ni los alumnos ni los docentes relacionen actualmente los contenidos en cuestión con el quehacer bioético, ni reflexionen activamente sobre sus cuestiones éticas en general. Es relevante destacar, no obstante, que los resultados de ACA develaron un tipo de información que permanecía oculta para Contreras Islas et al. (2016), y que resulta de gran utilidad para orientar futuras investigaciones relacionadas con la enseñanza de la bioética en la DCBS (por ejemplo, explorar la forma en que dichos elementos del contenido son tratados en la práctica docente y en el currículum oculto).

Si consideramos que el proceso de modificación de los planes de estudio a menudo responde a necesidades o exigencias de los gremios profesionales, los distintos valores de contenido dominante permiten, además, generar algunas hipótesis sobre la medida en que la bioética o la ética en general se consideran relevantes para el ejercicio de cada profesión. Por ejemplo, no es extraño que los planes de estudio más cercanos al área de las ciencias de la salud sean los que presentan valores de contenido general más elevados, hasta alcanzar un 3 en el caso de la licenciatura en medicina, para la cual el Consejo Mexicano para la Acreditación de la Educación Médica exige contenidos explícitos de bioética en el plan de estudios. Precisamente, en el área de la microbioética, propia del ejercicio estas profesiones, es donde la bioética ha tenido un desarrollo más intenso a nivel mundial durante los últimos 40 años (Actis y Outomuro, 2014; Callahan, 2015; Ruíz de ChávezGuerrero, 2015).

Finalmente, el contenido general permite establecer horizontes deseables para el contenido ideal de la bioética en los planes y programas de estudio -a partir, desde luego, del supuesto de que la incorporación de dichos contenidos al currículum de la DCBS resulta deseable-. Dicho valor ideal condensa aspectos cuantitativos y cualitativos, y es una ventaja de ACA sobre el análisis de contenido tradicional de Contreras Islas et al. (2016). Si bien es claro que los horizontes deseables deben ser elaborados de forma específica para cada plan de estudios, en un contexto histórico nacional y global como el presente las licenciaturas más próximas a las ciencias de la salud (Medicina, Enfermería, Estomatología, Química Farmacéutica Biológica y Nutrición Humana en Xochimilco, así como Biología Experimental en Iztapalapa) deberían contar con un contenido general cercano a 2.0. Por su parte, en las licenciaturas más cercanas a las ciencias biológicas (Agronomía, Biología y Medicina Veterinaria y Zootecnia en Xochimilco, así como Biología, Hidrobiología, las dos Ingenierías y Producción Animal en Iztapalapa) aspiraríamos a un contenido general aproximado de 1.0. En ambos casos, un contenido general cercano o igual a 0.0 constituye una deficiencia en el plan de estudios.

La realización del tipo de inferencias relacionadas en nuestro estudio con los índices de contenido general y contenido dominante -que resultan de gran utilidad para el análisis curricular e incluso para la planeación y el diseño de nuevos programas de estudio- constituye una de las ventajas de ACA frente a otras técnicas de análisis de contenido. Cabe reconocer, sin embargo, que ACA presenta al menos dos limitaciones importantes, que deben tomarse en consideración.

La primera limitación tiene que ver con la dificultad de automatizar los procesos de análisis para procesar grandes cantidades de información. Esta automatización es importante, por ejemplo, para procesar los 
contenidos que se generan constantemente en los medios digitales (Van Der Meer, 2016). Dado que las categorías más equívocas de ACA requieren considerar estructuras sintácticas, características semánticas e incluso el contexto de producción de los textos, sería necesario entrenar sistemas de machine learning para poder conducir un ACA automatizado. Esta dificultad de automatización, sin embargo, no es exclusiva de ACA, sino que se cuenta entre las desventajas más importantes de las técnicas existentes de análisis de contenido, las cuales consumen recursos temporales importantes (Bush et al., 2012).

Una segunda limitación, más profunda que la anterior, tiene que ver con el proceso de construcción de la escala ordinal de valores hermenéuticos, que, al quedar al criterio de los investigadores, podría afectar tanto la validez general como la reproducibilidad de los resultados y las inferencias que se realicen a partir de ellos. Debe considerarse en todo momento que la construcción de estas escalas implica en sí misma un proceso de interpretación respecto a la univocidad y la equivocidad de los procesos de interpretación subyacentes a los esquemas de codificación, lo que constituye una suerte de acto hermenéutico de segundo orden. Indagar sobre las implicaciones epistemológicas y filosóficas de esta limitación amerita un estudio más profundo y es una importante tarea pendiente.

\section{CONCLUSIÓN}

Visibilizar la naturaleza hermenéutica de las técnicas de análisis de contenido puede facilitar que los investigadores adopten una postura crítica respecto a la validez de sus categorías analíticas y los resultados que de ellas se obtuvieren. El análisis de contenido analógico (ACA) no solo visibiliza esta naturaleza, sino que la aprovecha para abrir nuevas posibilidades de inferencia. La principal novedad de ACA radica en la inclusión de una metacategoría, el valor hermenéutico, que permite organizar otras categorías analíticas o esquemas de codificación en una escala ordinal según su univocidad o equivocidad, indicando al mismo tiempo un distinto grado de validez para las interpretaciones que de ellas se obtuvieren. La construcción de dicha jerarquía se sigue directamente de los principios de la HA.

La posibilidad de hacer visibles diferentes formas en las que un mismo elemento está presente en las unidades de análisis confiere a ACA algunas ventajas sobre otras técnicas de análisis de contenido. Entre ellas, destaca el facilitar la relación con el contexto de producción de los textos, que ha sido uno de los mayores desafíos para las técnicas de análisis más tradicionales (Bush et al., 2012). A partir de la jerarquía de interpretaciones diferenciadas, ACA facilita la generación de hipótesis (e inferencias) respecto al contexto de producción de los textos, propiedad que resulta atractiva para distintos campos de investigación. El ejemplo desarrollado en las dos últimas secciones ilustra algunas de estas posibilidades para los casos específicos de la investigación educativa y los estudios curriculares, pero se espera que existan resultados semejantes en otros campos.

Para concluir, se desea destacar que el ejemplo de aplicación aquí presentado es apenas una muestra de la versatilidad de ACA, cuyas posibilidades se encuentran todavía muy poco exploradas. Por ello, se extiende la invitación a otros investigadores para explorar los alcances de esta técnica en otros campos de investigación.

\section{Agradecimientos}

Quiero agradecer a mi equipo de trabajo en la DCBS de la UAM Iztapalapa, y muy especialmente a los doctores Mauricio Beuchot de la Universidad Nacional Autónoma de México y Walter Beller de la UAM Xochimilco por su apoyo y orientación durante el desarrollo de la investigación de la que deriva el presente artículo. 


\section{REFERENCIAS}

Actis, A. M., y Outomuro, D. (2014). Bioética consolidada: abordaje histórido a más de 40 años de surgimiento. Revista de Bioética y Derecho, 30, 77-91.

Álvarez Díaz, J. A. (2015). Problemas éticos en reproducción humana. En O. López Arellano y F. Peña Saint Martin (Eds.), Salud, condiciones de vida y políticas sociales. Miradas sobre México (pp. 19-42). México: ALAMES PROMEP.

Beuchot, M. (2006). Exposición sucinta de una hermenéutica analógica. Communio, 39(2), 237-249.

Beuchot, M. (2007). Hermenéutica analógica y educación. Coahuila: Universidad Iberoamericana Torreón.

Beuchot, M. (2008). Perfiles esenciales de la hermenéutica. México: FCE, UNAM.

Beuchot, M. (2015). La hermenéutica y el ser humano. México: Paidós.

Bush, C., De Maret, P. S., Flynn, T., Kellum, R., Le, S., Meyers, B.,... Palmquist, M. (2012). Content Analysis. Recuperado de https://writing.colostate.edu/guides/guide.cfm?guideid=61 ( 9 de diciembre de 2018).

Bullen, M. (1998). Participation and critical thinking in online university distance education. Journal of Distance Education, 13(2), 1-32.

Callahan, D. (2015). Bioethics: its past and future. En G. Solinís (Ed.), Global Bioethics: What for? Twentieth anniversary of UNESCO's Bioethics Programme (pp. 19-23). Paris: UNESCO.

Conde Gaixola, N. (2001). Hermenéutica analógica, definición y aplicaciones. México: Primero editores.

Conde Gaxiola, N. (2010). La importancia de comprender la cultura del derecho a la luz de hermenéutica analógica. En N. Conde Gaxiola (Ed.), Hermenéutica Analógica y saberes sociales (pp. 207-224). México: Torres Asociados.

Contreras Islas, D. S., Lifshitz, C. K., Mendieta Márquez, E., y Pérez Hernández, M. A. (2016). Diagnóstico de componentes bioéticos en el ámbito educativo de Ciencias Biológicas y de la Salud. Revista Latinoamericana de Bioética, 17(1), 14-35. doi: http://dx.doi.org/10.18359/rlbi.2137

Domas White, M., y Marsh, E. E. (2006). Content Analysis: A Flexible Methodology. Library trends, 55(1), 22-45.

Elo, S., y Kyngäs, H. (2007). The qualitative content analysis process. Journal of Advanced Nourcing, 62(1), 107-115.

Galindo González, J. (2010). Hacia una heurística analógica en las Ciencias Sociales. En N. Conde Gaxiola (Ed.), Hermenéutica Analógica y saberes sociales (pp. 69-164). México: Torres Asociados.

González Valenzuela, J. (2008). ¿Qué ética para la bioética? En J. González Valenzuela (Ed.), Perspectivas de bioética (pp. 9-50). México: Fondo de Cultura Económica, UNAM, Comisión Nacional de Derechos Humanos.

González Valenzuela, J., y Linares Salgado, J. E. (2013). Diálogos de bioética. Nuevos saberes y valores de la vida. México: Fondo de Cultura Económica, UNAM.

Grondin, J. (2002). Introducción a la hermenéutica filosófica. Barcelona: Herder.

Grondin, J. (2008). ¿Qué es la hermenéutica? Barcelona: Herder.

Neuendorf, K. A. (2002). The content analysis guidebook. Thousand Oaks: Sage.

Palazón Mayoral, M. R. (2014). Sugerencias hermenéuticas para la educación. Perfiles educativos, XXXVI(146), 174-186.

Ricœur, P. (1986). Du texte à l'action. Paris : Du Seuil.

Rivero Weber, P. (2006). El papel de la hermenéutica en la docencia. En M. Beuchot y A. Velazco Gómez (Ed.), Hermenéutica, discurso y sociedad. Sextas jornadas de hermenéutica (pp.73-80). Mexico: UNAM.

Rourke, L., y Anderson, T. (2004). Validity in Quantitative Content Analysis. Educational Technology Research \& Development, 52(1), 5-18.

Ruiz de Chávez-Guerrero, M. H. (2014), Veinte años de bioética en México: desarrollo y perspectivas de la Comisión Nacional de Bioética. Cirugía y cirujanos, 82(6), 699-708.

Sagols, L. (2013). La ética ante la crisis ecológica. México: Fontamara, UNAM.

Sagols, L. (2015). The bio-philia future of bioethics. En G. Solinís (Ed.), Global Bioethics: What for? Twentieth anniversary of UNESCO's Bioethics Programme (23-26). Paris: UNESCO. 
Solan, A., \& Bowe, B. (2014). Phenomenology and hermeneutic phenomenology: the philosophy, the methodologies, and using hermeneutic phenomenology to investigate lecturers' experiences of curriculum design. Quality \& Quantity, 48, 1291-1303.

Van der Meer, T. (2016). Automated content analysis and crisis communication research. Public Relations Review, 42, 952-961.

Velazco Gómez, A. (2006). Hermenéutica y multiculturalismo. En M. Beuchot y A. Velazco Gómez (Ed.), Hermenéutica, discurso y sociedad. Sextas jornadas de hermenéutica (pp. 143-149). México: UNAM.

\section{Notas}

1 Aspecto que, para algunos autores (Bush et al., 2012; Neuendorf, 2017), figura entre las principales limitaciones o desventajas de para las técnicas de análisis de contenido.

2 Cabe señalar que, a partir del trabajo de Ricœur, la noción de texto alcanza una amplitud casi infinita que comprende todo aquello que es susceptible de ser comprendido, con lo que la hermenéutica alcanza el estatus de filosofía universal de la comprensión (Grondin, 2008; Ricœur, 1986).

3 Con apoyo en el trabajo de Diego Gracia y Adela Cortina, Álvarez (2015) propone distinguir tres niveles de la bioética: la microbioética, que se ocupa de la toma de decisiones en torno al propio cuerpo, especialmente al nivel de la clínica; la mesobioética, que remite a la toma de decisiones institucionales y estructurales en el ámbito de la salud, y, finalmente, la macrobioética, que abarca cuestiones de ecoética, justicia global y biopolítica (Álvarez, 2015, pp. 25-26)

4 El concepto de unidades de enseñanza-aprendizaje (UEA) se introdujo en el discurso institucional de la UAM con el objetivo de poder abarcar tanto las asignaturas de los sistemas de enseñanza tradicionales, propios de las unidades Iztapalapa y Azcapotzalco, como los módulos del sistema modular (valga la redundancia) de la unidad Xochimilco. Así pues, en el caso de Iztapalapa las UEA comprenden asignaturas independientes dentro de un mismo plan de estudios, mientras que en el caso de Xochimilco se refieren a módulos interdisciplinarios y trimestrales.

5 Esta visión de responsabilidad social quedó recogida en los artículos 2 y 3 de la Ley Orgánica de la UAM, que puede descargarse del sitio web de la universidad (http://www.uam.mx).

Figura 1Esquema de la organización en metacategorías empleando el valor hermenéutico. En (A) el valor hermenéutico (vh) se usa para organizar las categorías analíticas (CA) de acuerdo con su validez. En (B) el valor hermenéutico (vh) se emplea para distinguir la univocidad o equivocidad de distintos esquemas de codificación (Cod) dentro de cada categoría analítica (CA). Fuente: elaboración propia, 19 de diciembre de 2018.

\section{BY-NC-SA}

